\title{
Aristotle vs. Ringelmann \\ On Superlinear Production in Open Source Software
}

\author{
Thomas Maillart* \\ University of Geneva, Switzerland \\ Didier Sornette† \\ ETH Zurich, Switzerland
}

(Dated: April 17, 2019)

Organizations exist because they provide additional production gains, in comparison to horizontal ways of allocating resources, such as markets [1], and the open source movement is deemed to be a new kind of peer-production organization somehow in between hierarchically organized firms and markets [2]. However, to strive as a new kind of organization, open source must provide production gains, which in turn should be measurable. The open source movement is particularly interesting to study for this reason. Here, we confront and discuss two contrasting views, which were reported in the literature recently. On the one hand, Sornette et al. [3] uncovered a superlinear production mechanism, which quantifies Aristotle adage: "the whole is more than the sum of its parts". On the other hand, Scholtes et al. [4] found opposite results, and referred to Maximilien Ringelmann, a French agricultural engineer (1861-1931), who discovered the tendency for individual members of a group to become increasingly less productive as the size of their group increases [5]. Since Ringelmann, the topic of collective intelligence has interested numbers of researchers in social sciences and social psychology [6], as well as practitioners in management aiming at improving the performance of their team [7]. In most research and practice case studies, the Ringelmann effect has been found to hold, while, in contrast, the superlinear effect found by Sornette et al.is novel and may challenge common wisdom [3]. Here, we compare these two theories, weigh their strengths and weaknesses, and discuss how they have been tested with empirical data. We find that they may not contradict each other as much as was claimed by Scholtes et al. [4]. 


\section{INTRODUCTION}

In psychology (Gestalt theory [8]), biology (brain functions [9], ecological networks [10]), physics (spontaneous symmetry breaking [11] and the "more is different" concept [12]), and in economics [13, 14], the famous adage by Aristotle "the whole is more than the sum of its parts" has inspired research in complexity science, in particular regarding emerging behaviors in nature and society [15, 16]. Indeed, the raison d'être of societies is the prospect that people will achieve more together, yet at some individual alienation costs [17]. For a society to strive, these alienation costs should overall be smaller than the benefits a society can bring to its members. Ideally, fair distribution of benefits should be organized through institutions [18] that implement robust mechanisms to enforce cooperation [19, 20].

One special instance of a society is the firm. A firm is an organization devoted to production, which is born from the internalization of transaction costs associated with gathering production resources, in particular human resources: at some point it is less costly to permanently hire an individual whose skills are needed often than sourcing them repeatedly on a market [1]. Hence, the employee enters a permanent contractual relation and thus, an organizational structure at some alienation costs (less freedom to contract with other parties).

The open source movement operates in a slightly different fashion: peer-production [2] prescribes that participants to an open source project mainly obey two rules : (i) task self-selection and (ii) peer-review. In a nutshell, contribution (i.e., production) enforcement mechanisms are very loose, neither relying on hierarchical organization nor market mechanisms and there is no clear counterpart to contributions in open source development. The lack of explicit organization rules in open source has generated much attention in management science [21, 22], complex systems and network dynamics [23], law and economics [24, 25], with one overarching question being how self-organized communities gather and, moreover, produce efficiently together in absence of organizational rules clearly tied to incentives [26].

*Electronic address: thomas.maillart@unige.ch

${ }^{\dagger}$ Electronic address: dsornette@ethz.ch 
The open source movement gathers people with heterogeneous incentives, ranging from hedonism to paid jobs [26]. It is therefore difficult to measure the implications of individual, and of collective intelligence and coordination, on the production of source code. In particular, there is the question of how cumulative innovation emerges from self-selected contributions and peer-review, which on average make software more robust and help the emergence of new functionalities. Measuring production and productivity of collective intelligence may be a significant addition to the debate, and attempts to measure productivity of software developers is nearly as old as the software industry [27], with several models developed to measure the efficiency of software programmers, yet with the assumption that programmers work in a corporate environment, which is usually highly scheduled.

The bottom-up and collective intelligence aspects of production have been much less covered, in open source and more generally, in open collaboration [28]. Dealing with groups such as firms and production units, management science also aims to understand when and how a group can produce more than the sum of its individual contributions, and to design ways to improve team performance [29 32], through the mechanism of complementarity in organization [33, 34] and innovations [35]. Because most activities in our modern environment require coordination and collaborative actions within groups of widely varying sizes, it is the fundamental aspiration of any manager, be it in the public or private sector, to find and master the determinants of enhanced productivity. Since Ringelmann, the topic of collective intelligence has interested numbers of researchers in social sciences and social psychology [6], as well as practitioners in management aiming at improving the performance of their team [7].

Despite their conflicting views, the contributions by Sornette et al. [3] and Scholtes et al. 44] provide key insights on that matter, in particular, yet not limited to, open source development. We focus on these two papers in particular because Scholtes et al. [4] challenged evidence brought forth by Sornette et al. [3], creating confusion or perhaps even worse the sentiment that the superlinear productivity law is stillborn, having been killed just out of its academic womb. In the remaining of this paper, we describe and compare the two approaches (Section【I), then discuss the strengths and weaknesses of each approach 
(Section III), and conclude (Section IV).

\section{PRODUCTION AND PRODUCTIVITY MEASURES FOR OPEN SOURCE SOFTWARE}

Here, we expose the two contrasting perspectives taken by Scholtes et al. [4] on the one hand, and by Sornette et al. [3] on the other hand.

\section{A. The Ringelmann effect in software engineering}

There is a common wisdom supported by a vast majority of studies, which tend to show that teams of software developers become less productive as they get bigger. In empirical software engineering, this phenomenon is known as the Brooks law of software project management, which states that "adding manpower to a late software project makes it later" [27]. The identified cause for the Brooks' law is the increasing coordination costs involved as teams get larger. In social psychology, this phenomenon is also known as the Ringelmann effect, in reference to Maximilien Ringelmann, a French agricultural engineer (1861-1931) who discovered the tendency for individual members of a group to become increasingly less productive as the size of their group increases [5].

Scholtes et al. 44] performed a study using a dataset of 58 open source software (OSS) projects, which amount in total to more than 580,000 commits contributed by more than 30,000 developers. Their study indeed finds that the Ringelmann effect seems to hold on average. Here is the way they proceeded for their study. While in structured organizations, a team can be easily defined and measured, a team in OSS projects is more complicated. Indeed, Scholtes et al. [4] reported that $40 \%$ of contributions to OSS projects (i.e., commits) were made by one-time contributors. Researchers have identified different circles of contributors from a core team (producing up to $90 \%$ of the source code), to less involved contributors, to one-shot contributors, and finally, to lurkers, who follow the advancement of a project without contributing to the source code, and yet participating e.g., on the mailing list or posting issues [36]. The heterogeneous, distributed, and uneven proportion of contributions makes the study of OSS project organizations complicated, 


\begin{tabular}{|l|c|c|}
\hline & Scholtes et al. & \multicolumn{1}{c|}{ Sornette et al. } \\
\hline OSS Projects studied & 56 & 164 \\
\hline Number of Contributors & 30,845 & 15,650 \\
\hline Number of Commits & 581,353 & $8,220,673$ \\
\hline Project sampling & $\bullet>1$ year of activity & $\begin{array}{l}\text { Random sampling : Power } \\
\text { law distribution found } \\
\text { Pr }(\text { size }>S) \sim 1 / S^{1.4} \text { with } \\
S \text { the number of contributors }\end{array}$ \\
\hline
\end{tabular}

TABLE I: Comparison of datasets used by Scholtes et al, and Sornette et al.

particularly across projects, themselves of heterogeneous nature.

Scholtes et al. provided a dynamic formulation of what a team is, considering that a developer, who has not contributed after 295 days has $10 \%$ chance to further contribute. Therefore, they chose a window of 295 days to define a team size at time $t$, which is the count of contributors who have committed at least once in the last 295 days. The output must also be measured. Various measures of source code production have been developed to account for contribution effort [37, 38]. Scholtes et al. decided to focus on quantifying changes, as measured by the edit distance (also called the Levenshtein distance [39]), i.e., the minimum number of bytes one has to permute/add/delete to compare between a version of the source code and a committed update. Scholtes et al. used averaged contributions over time windows of 7 days (rationalized by the fact that in $90 \%$ of the cases, two consecutive commits occur within this time window).

Scholtes et al. first measured the output (i.e., number of commits and contributions as defined by edit distance over the last 7 days) as a function of the input (i.e., active developers within the same 7 days window at time $t$ ). They found that, when the number of developers increase, the mean contribution per developer decreases. Moreover, when considering mean contribution per active developer as a function of team size (i.e., developers active in the last 
295 days), the results show the same negative scaling function [for commits : $\sim$ team ${ }^{-0.24}$ $\left(p<0.001\right.$ and $\left.r^{2}=0.16\right)$ and for contributions $\sim$ team ${ }^{-0.36}\left(p<0.001\right.$ and $\left.r^{2}=0.08\right)$, obtained by MM-estimation]. The authors highlight the particularly low $r^{2}$ in both case, reflecting the high variability of their average measures, and as such, they conclude that it is impossible to make robust predictions from these scaling laws. Considering the output as a function of team size (here, the input is considered as the amount of resources available, i.e., contributors who have roughly more than a $10 \%$ chance to contribute), again negative scaling properties are found [for commits : $\operatorname{team}^{-0.75}\left(p<0.001\right.$ and $\left.r^{2}=0.44\right)$ and for contributions : team ${ }^{-0.86}\left(p<0.001\right.$ and $\left.r^{2}=0.25\right)$, obtained by MM-estimation].

As a consequence, Scholtes et al. concluded that "OSS communities are indeed no magical exception from the basic economics of collaborative software engineering", and they further attempted to substantiate the observed decreasing return on scale, considering two commonly accepted reasons for the Ringelmann effect : (i) free-loading and (ii) coordination costs. They concentrated on the latter because there is a substantial body of evidence and research work on coordination in software engineering. Although the authors did not mention it, it is indeed hard, if not impossible, to define free-loading when contributors are actually not compelled to contribute (following the general rules of peer-production applicable in OSS). To assess coordination effort and its effects on productivity, Scholtes et al. borrowed from Cataldo et al. [40] and computed the co-edition directed network for all developers (direction stands for chronological influence) as a function of time (i.e., time windows of 7 days), with a distinction between out-degree $k_{\text {out }}$ (i.e., one developer has to build on changes by $k_{\text {out }}$ other developers) and in-degree $k_{\text {in }}$ (i.e., $k_{\text {in }}$ developers must build on changes by one developer). Scholtes et al. considered first the mean out-degree as a function of the size of the coordination network [69]. The mean out-degree and the size of the coordination network seem to be positively correlated, but it is not clear what we can learn from this result (Figure 11 in Scholtes et al. [4]; not discussed in the paper). Finally, Scholtes et al. considered mean out-degree as a function of the negative productivity scaling exponent described above. They found that projects with "strongly negative and significant slopes for the scaling of productivity also exhibit pronouncedly positive scaling exponents for the growth of the mean (weighted) out-degree" (Figure 12 in Scholtes et al. [4]). 


\begin{tabular}{|l|c|c|}
\hline & Scholtes et al. & Sornette et al. \\
\hline Activity window & 7 days & 5 days \\
\hline Team definition & $s:=$ at least one contribution in & all developers \\
\hline last 295 days & within 7 days windows & $c$ (within 5 days windows) \\
\hline Primary production & commit contributions (Levenshtein & $R:=$ number of commits \\
\hline Productivity & distance between commits) & number of commits $R$ \\
performed by $c$ active \\
properties
\end{tabular}

TABLE II: Comparison of definitions and results by Scholtes et al., and Sornette et al. Comparing both approach, we observe that resorting to averaging (Scholtes et al.) leads to tremendously different results, compared to a direct measure of production per developer (Sornette et al.).

Based on these results, Scholtes et al. asserted that OSS projects exhibit dis-economies of scale in production as a function of team size, and hence, sub-linear productivity. They rejected the evidence that "the whole is more than the sum of its parts" evidenced by the superlinear productivity shown by Sornette et al. [3].

\section{B. The Aristotle effect perspective}

In contrast to the previous section, by analyzing 164 open source software (OSS) projects of broadly distributed sizes ranging from 5 to 1,678 contributors, Sornette et al. [3] found that contribution activity $R$, defined in terms of number of commits, within a time window of 5 days, is a superlinear function $R \sim c^{\beta}$ of the number of active developers $c$ during the same period. The superlinear exponent is on average $\hat{\beta} \approx 4 / 3$, over all projects studied, with a rather large variability with $\hat{\beta}$ ranging from 1 to 3 . They found that $\hat{\beta}$ tends to 
decrease with the number of contributors in the five day window, fluctuating around 1 or less for more than 30 to 50 contributors. Moreover, as reported in Sornette et al. [3], the distribution of total number of developers per project is heavy-tailed, i.e., with many small projects and a few very large ones.

Sornette et al. explored two possible mechanisms generating the observed superlinear phenomenon : (i) an interaction-based mechanism (including interactions leading to a phase transition or to a super-radiance phenomenon [41]) and (ii) a large-deviation mechanism, based on the fact that, in the presence of a heavy-tailed distribution of contributors per project, many developers contribute just few commits while a minority contribute most of the commits; then, the larger the group size, the more likely it is for a large contributor to be present, leading to the superlinearity phenomenon. The observation that a few developers dominate the overall contribution is well-known in OSS, and is also reported by Scholtes et al. Sornette et al. did not attempt to distinguish which one of these two mechanisms might be at work. They however considered that both the interaction-based and the large deviations mechanisms can be captured together by a generic cascade process, which has been found to be well described by self-excited Hawkes conditional Poisson processes [42], in particular for human dynamics [43 47], taking into account the specifics of human timing [48].

The Hawkes process is defined by the conditional point process intensity $I(t)$ of events (commits) given by

$$
I(t)=\lambda(t)+\sum_{i \mid t_{t}<t} f_{i} \phi\left(t-t_{i}\right),
$$

where $\left\{t_{i}, i=1,2, \ldots\right\}$ are the timestamps of past commits, $\lambda(t)$ is the spontaneous exogenous rate of commits, $f_{i}$ is the fertility of commit $i$ that quantifies the number of commits (of first generation) that it can potentially trigger directly, and $\phi\left(t-t_{i}\right)$ is the memory kernel, whose integral is normalized to 1 , which weights how much past commit activities influence future ones. According to (1), the number of commits contributed between time $t$ and $t+d t$ results from two sources: (i) an exogenous source $\lambda(t) d t$ representing the spontaneous commits not related to previous commits; (ii) an endogenous term represented by the sum over all commits that were made prior to $t$, and which are susceptible to trigger 
future commits. The class of Hawkes models can be mapped onto the general class of branching processes [49]. The statistical average fertility $\left\langle f_{i}\right\rangle$ defines the branching ratio $n$, which is the key parameter. For $n<1, n=1$ and $n>1$, the process is respectively sub-critical, critical and super-critical [50, 51]. Interpreting a cluster or connected cascade in a given branching process of triggered contributions as the burst of production in a group of developers, the distribution of contributions is thus mapped onto that of triggered cluster sizes [52].

Sornette et al. found and empirically validated that, at criticality, there is a relationship between the power law tail distribution (with exponent $\gamma \approx 1.5$ ) of activity per contributors per time bin of 5 days, the power law tail distribution of cluster size, which is equivalent to the production $R$ per contributor with renormalized exponent $\mu=1 / \gamma$ and the superlinear scaling exponent $\beta=\gamma=1 / \gamma$. However, as already mentioned, Sornette et al. found that the superlinear scaling exponent $\beta$ tends to decrease as a function of the total number of contributors in an OSS project. Likewise, the frequency of productive bursts is reduced for larger projects, suggesting that large projects bear additional coordination costs.

\section{ARISTOTLE VERSUS RINGELMANN ?}

Before considering the fundamental differences between the two approaches presented here, and their validity, we shall highlight some results, which to some extent bear resemblance. Sornette et al. found that large projects tend to exhibit less powerful and less frequent superlinear productive bursts. This result may look similar to the findings by Scholtes et al., who studied only large projects. However, Sornette et al. do not say that there are dis-economies of scale, but rather that economies of scales appear to be weaker. Similarly to the co-edition directed network model developed by Scholtes et al., the self-excited Hawkes conditional Poisson process measures how past commits influence future commits. It does so in a way that incorporates the influence all past events, while the network approach by Scholtes et al. relies only on 7-day contribution windows. In other words, Scholtes et al. considered that, in order to be dependent (and bear coordination costs between each other), two commits must occur within the same time window. The network approach, taking into account who changed which file, brings more information 
regarding how contributions relate to each other. It is also interesting to note the closeness of the short- and long-term time windows used in the two studies : 7 and 295 days for Scholtes et al. versus 5 and 250 days for Sornette et al. While Scholtes et al. provide a justification, Sornette et al. are only concerned with robustness and check that the same results are obtained by varying the short-term window. In Sornette et al., no rationale is provided for the long-term window.

Beyond these resemblance and arguably a common research question, nearly every other aspects differ in the two studies: the chosen approach, the definitions of productivity, and the data used. This raises a number of questions on the main claim by Scholtes et al. that Sornette et al. were wrong about the superlinear productive bursts [4]. In the following, we thus highlight and discuss these methodological divergences. Moreover, we discuss the approach chosen by Scholtes et al. to take on the results by Sornette et al, in an era that promotes open science and, most importantly, reproducibility of scientific results.

\section{A. Productivity \& Team Size}

We start by a fundamental conceptual remark that illuminates one key difference between the approach of Scholtes et al. [4] and the one by Sornette et al. [3]. Scholtes et al. consider production in the mean, using as metric the average output per team member (Introduction, 2nd paragraph, line 3), and argue that it increases when synergy effects are present and decrease due to communication and coordination overhead (which surges with larger teams). In contrast, Sornette et al. argued and demonstrated that using an average output is misleading in the presence of highly bursty dynamics characterized by power law tail distributions with small tail exponents. This empirical fact is also cited by Scholtes et al. and well-documented in the open source software production literature and for other open collaboration projects, such as wikis and Wikipedia [53 56]. In open collaboration, a few contributors account for a majority of performed work, whether counted in lines of code, commits, files modified, and so on. This is one of the features associated with the fact that the distribution of contributions, counted in commits or in lines of codes, possesses a power law tail of the form $P(X>x) \sim 1 / x^{\mu}$ with $\mu<1$ [3]. Such distributions are wild [57] in the sense that their two first statistical moments (mean and variance) are undefined 
and diverge as the sample grows. For such heavy-tail distributions, reasoning in mean is fundamentally erroneous, as Scholtes et al. could indeed experience when trying to perform predictions (c.f., Section 4.1 in [4]). For a finite number $n$ of developers in the project, it is easy to show that the average production scales as $\sim n^{1 / \mu}$ for $\mu<1$ and $\sim n$ for $\mu \geq 1$. Defining productivity as the ratio of the total production by the number $n$ of team members, this shows that productivity scales as $\sim \frac{n^{1 / \mu}}{n}=n^{\frac{1}{\mu}-1}$ for $\mu<1$ and is constant for $\mu \geq 1$. This latter case is the null hypothesis of an approximate constant output per team member. Superlinear production is quantified by $\mu<1$, leading to a growing productivity per team member, the larger the team. Searching for a superlinear productivity is different from seeking a superlinear production, the former requiring $\frac{1}{\mu}-1>1$, i.e., $\mu<1 / 2$, while the later just needs $\mu<1$. In their dataset of 164 projects, Sornette et al. found that only four projects are characterized by $\mu<0.5$ (while most obey $\mu<1$ ). Scholtes et al. have used a much smaller dataset of 58 projects, which implies a $\approx 0.8 \%$ chance to find one project for which the average productivity scales superlinearly with team size.

More generally, the definition of productivity needs to be carefully addressed. Indeed, an open source software community does not come into being fully grown. It starts rather small and then grows progressively - one could say organically - with the project. When growing, the community bears increasing communication and coordination costs as pointed out by Scholtes et al. While recognizing the importance of different team sizes, Scholtes et al., picked projects meeting the following criteria: (i) at least one year of activity, (ii) 50 different active developers, and (iii) being among the 100 most popular projects, as measured by the number of forks on GitHub, a leading online service for open source software production. In contrast, Sornette et al. chose a representative sample of the open source ecosystem with 134 projects with less than 50 developers and 30 projects with more than 50 developers (with a minimum of 5 developers). The representative sampling of projects (see Figure 1 in [3]) showed that the superlinear production is usually valid only for projects of sizes no more than 30 to 50 members who are active at a given time. Sornette et al. found statistically significant evidence that the superlinear production tends to fade away to just linear production (i.e., constant productivity per developer) for projects with more than 50 developers (see Figure 8 in [3] ]). In other words, the sample selection made by Scholtes et al. seems heavily biased towards 
large projects, which represent the few large (presumably older) projects and are indeed exposed to more communication and coordination costs, and also exhibit less synergy effects.

More specifically, Scholtes et al. defined a team as the set of developers who are active at least once within a time window of 295 days, determined by the 90th quantile of the distribution of times between two consecutive commits by the same person. This definition excludes developers with a unique contribution, who nevertheless account for $40 \%$ of all contributions, as reported by Scholtes et al. in Section 3.2 of [4] (end of second paragraph). In line with our above remarks concerning the heavy-tailed distribution of contribution sizes, this definition amounts to throw away the baby with the bath, since it is fundamentally ill-suited to account for the fact that a few, often most senior, developers may not contribute for years in between two commits (see Figure 2 in [58]), while at the same time they may account for most of the contribution production. The definition of contributors proposed by Scholtes et al. is thus biased with respect to the special nature of the open source software community, which is, almost by essence, different from a corporate organization, as documented in a number of management science articles (see e.g., [26] and references therein).

Yet, productivity may be defined in a variety of ways, each with their advantages and shortcomings. Scholtes et al. considered productivity as production per active developers among a team (defined as an aggregate of working developers in large - 295 days - time windows), while Sornette et al. considered productivity as production per developer and per time unit (i.e., over a short time period of 5 days). Even though not perfect, the latter definition is more fine-grained than the one proposed by Scholtes et al., and precisely allows capturing the subtle highly non-linear bursts of activity reported in [3], which could not be observed by averaging developer engagement (over a team aggregate and over time). In essence, two visions oppose each other: Scholtes et al. adopted a software engineering perspective, which takes roots in the necessity to measure the effort and productivity by software developers in a competitive industry. Because of the complexity of information systems, and the importance of outsourcing, the software development industry may suffer from the principal-agent problem [59] and hence, may require controlling. The software engineering perspective is reflected by the sampling of only large projects (suggesting 
that smaller projects are not really worth studying), and by the definition of active contributors and teams, which ignores $40 \%$ of the contributors. On the contrary, Sornette et al. considered the OSS ecosystem with no filter, taking a more general approach in project sampling, in the definition of contributions, and in theory elaboration and validation.

\section{B. Commits \& superlinear scaling}

Productivity is the ratio of an output and an input. So far, we have mainly discussed the developer input, i.e., the human capital. Scholtes et al. raised concerns about the output, and claimed that the number of commits is an erroneous measure of production. For that, they bring forth the following argument: the total number of commits contributed by $n$ developers active in a given time period cannot - by definition - be less than $n$, which is why the total number of commits must scale at least linearly with team size. This apparently common-sensical claim is incorrect as we demonstrate here. Let us consider $n$ developers. The largest contributor makes $N$ commits (resp. lines of code). The second one contributes $N / 2^{\alpha}$ commits. The third one contributes $N / 3^{\alpha}$ commits, and the $n$-th one contributes $N / n^{\alpha}$ commits. If $0<\alpha<1$ and $n$ and $N$ are such that $N / n^{\alpha} \geqslant 1$ (i.e., $n \leqslant N^{1 / \alpha}$ ), then the total number of commits contributed by $n$ developers is given by

$$
S(n)=\frac{N}{1^{\alpha}}+\frac{N}{2^{\alpha}}+\frac{N}{3^{\alpha}}+\cdots+\frac{N}{j^{\alpha}}+\cdots+\frac{N}{n^{\alpha}} \sim N \cdot n^{1-\alpha} .
$$

Thus, in this example, the total contributions of these developers grow sub-linearly as a function of group size $n$, with exponent $1-\alpha$. Let us illustrate this demonstration by a numerical example, showing that the sublinear effect is clearly visible even for small team sizes. Let us assume that $N=10$ and $\alpha=1 / 2$. For $n=5$ developers, the total number of commits is 32 . For $n=25$, the total contribution is equal to 86 commits, which is 2.7 times that for the team of 5 developers (and not 5 times more). Note that for the team of 25 developers, the first contributor makes 10 commits and the last one contributes 2 commits. We believe Scholtes et al. made a very common confusion between absolute numbers and scaling properties. More generally, in the field of fractals, this error is also often found in the literature that confuses the fact that the fractal dimension (here, the scaling exponent) tells nothing (or very little) about the density (here, the number of commits per developer). 
Dismissing commits as a measure of production, Scholtes et al. used the Levenshtein edit distance [39] of source code changes between two consecutive commits (i.e., so-called diffs). The Levenshtein edit distance counts the number of permutations, additions and deletions of characters necessary to match two different strings. Using the Levenshtein edit distance is without doubt more detailed, but it is not sufficient to dismiss commits. Even though they have not used the Levenshtein edit distance, Sornette et al. showed that superlinear scaling production holds as well for lines of code (see Figure 3 in [3]), up to an additional scaling factor that defines the relation between commits and lines of code. In order to properly dismiss commits as a measure of contribution, Scholtes et al. may have wanted to show that there is no relation between commits and their contribution metric, for which there is no clear consensus in the scientific literature. The Levenshtein distance is more detailed than commits, but may not necessarily contain additional relevant information. Moreover, at a qualitative level, we should stress that using the more detailed Levenshtein edit distance is not without its own problems. One may indeed argue that changing one character or a single line of code in a piece of software, while quantified as minor by the Levenshtein distance, could be in some cases a tremendous output reflecting a major commitment in terms of human capital (think e.g., of a small edit correcting a security vulnerability) [60 62]. We suggest that a truly faithful measure of input would be the time effectively spent in front of a computer by a contributor in order to achieve a task for the focal open source software project. Unfortunately, this information is not available to open source software researchers and, even if it would be available, one could endlessly debate on a broad (resp. narrow) definition of time consumption, and whether the coffee break and the ping-pong sessions are actually parts of the production time: nearly all Silicon Valley software companies would include this time as truly productive time. Another way of proceeding would be to use a robust approach to attribute value to each contribution instead of assuming value. Such an approach to attribute value to contributions has been previously proposed by Maillart et Sornette [63]. In more conditioned environments, other ways to attribute contribution value to individuals engaged in collective intelligence have been tested and studied [64]. 


\section{From Aristotle to Ringelmann : a missed opportunity for reproducible science ?}

Open science is nowadays highly promoted to ensure reproducibility of scientific results, and to encourage research groups to "debug" and build upon each other works [66, 67]. The open science movement is inspired by the open source software movement, best summarized by the seminal adage: Given enough eyeballs, all bugs are shallow [65]. The authors of the paper From Aristotle to Ringelmann: a large scale analysis of team productivity and coordination in Open Source Software projects are (or were) members of the Chair of Systems Design. The Chair of Systems Design has been known to be a pioneering research group at ETH Zurich, advocating the use of open source software and contributing significantly to the open access movement. Sornette et al. published in PlosOne, which the first and leading open access scientific journal. Along with the paper, they submitted and shared the data they used for their study. Scholtes et al. clearly framed their paper as a response to Sornette et al. ("From Aristotle to Ringelmann" in the title), and they claimed that the results published by Sornette et al. do not hold. The claim by Scholtes et al. is at best misleading as they did not bind to elementary principles of science reproducibility. First, they neither used the same data nor detailed the potential implications of using a different dataset. Second, they neither invalidated the method by Sornette et al. nor compared thoroughly both approaches, with their pros and cons, as we have done above. Third and foremost, they did not bring compelling arguments for changing the assumptions underlying the analyses. These limitations deeply undermine their claims that the results of Sornette et al. are incorrect, as we have shown above. As a result, it is challenging to weigh the value of one approach against the other, and in this regard, limits the pertinence of the contribution by Scholtes et al.

Scholtes et al. submitted to and published their paper in the Journal Empirical Software Engineering. First, one would expect that claims questioning the validity of the results obtained by Sornette et al. should have been sent to the same journal (i.e., PlosOne), as a comment or a follow-up paper to the editors. Second, it is rather surprising that the editors and the reviewers of the Journal Empirical Software Engineering did not raise any issue concerning the approach by Scholtes et al. to rebut the findings by Sornette et al., in particular given the many problems that we have highlighted above. Third, when the 
present authors attempted to send an earlier version of this manuscript [68] as a response to the editor, they received the following response from the editors of the Journal Empirical Software Engineering: "the [...] journal does not publish any responses to articles. We encourage you to expand the response to a full research paper, e.g., by repeating the experiments, adding additional research questions, etc". In other words, the editors barred the possibility to react to Scholtes et al. in their journal, and they asked the present authors to perform what they should have requested at first for the manuscript by Scholtes et al.

\section{CONCLUSION}

We have carefully described the two methods and results by Sornette et al. [3] and Scholtes et al. [4], and their apparent opposite results (i.e., the Aristotle vs. Ringelmann effects), with emphasis on their commonalities and differences. Despite claiming that the results by Sornette et al. do not hold, Scholtes et al did not use the same data (made available by Sornette et al. following open access standards), they used a totally different methodology (based on averages) that does not allow a direct testing of the methods and results by Sornette et al. (designed to be able to quantify bursty dynamics and large deviations). However compelling and probably valid in its own way, the method followed by Scholtes et al. does not help directly invalidate the results and theory by Sornette et al. We believe there is much room for the Aristotle vs. Ringelmann debate, and we are glad that Scholtes et al. took upon the challenge. Our conclusion is that Sornette et al.'s results hold for no more than 3050 contributors working simultaneously, while Scholtes et al.'s results may apply for larger projects. Yet, we believe that proceeding in a way that follows good practices regarding open and reproducible science, as well as using a more standard publication channel for their challenge, would have helped developing a much more data grounded, constructive and serene debate. 


\section{Acknowledgements}

Thomas Maillart acknowledges support from the Swiss National Science Foundation (grants P3P3P2_167694 and P300P2_158462).

[1] Coase RH The Nature of the Firm, Economica 4: 386-405 (1937).

[2] Benkler Y, Coase's Penguin, or, Linux and "The Nature of the Firm", The Yale Law Journal 112: $369+(2002)$.

[3] Sornette, D., Maillart, T. and Ghezzi, G., How Much Is the Whole Really More than the Sum of Its Parts? 1? 1= 2.5: Superlinear Productivity in Collective Group Actions. Plos one 9.8, e103023 (2014).

[4] Scholtes, I., Mavrodiev, P. and Schweitzer, F. , From Aristotle to Ringelmann: a large-scale analysis of team productivity and coordination in Open Source Software projects, Empir. Software Eng., 21, 642-683 (2016).

[5] Ringlemann, M., Recherches sur les moteurs animés: Travail de l'homme, Annales de l'Institut National Agronomique. 121, (1913).

[6] Woolley, A. W., Chabris, C. F., Pentland, A., Hashmi, N., and Malone, T. W. Evidence for a collective intelligence factor in the performance of human groups. science, 330(6004), 686-688, (2010).

[7] Woolley, A. W., Aggarwal, I., and Malone, T. W. (2015). Collective intelligence in teams and organizations. Handbook of collective intelligence, MIT Press, 143-168.

[8] Humphrey, G., The Psychology of the Gestalt, Journal of Educational Psychology 15: 401 (1924).

[9] Damoiseaux, J., Greicius, M.,Greater than the sum of its parts: a review of studies combining structural connectivity and resting-state functional connectivity. Brain structure \& function 213: 525-533 (2009).

[10] Jorgensen, S.E. Introduction to Systems Ecology (Applied Ecology and Environmental Management). CRC Press (2012).

[11] Anderson, P.W. Plasmons, Gauge Invariance, and Mass. Physical Review Online Archive (Prola) 130 439-442 (1963). 
[12] Anderson, P.W. More Is Different. Science 177 393-396 (1972) .

[13] Arthur, W.B. Increasing Returns and Path Dependence in the Economy (In Economics, Cognition, and Society), University of Michigan Press (1994).

[14] Krugman, P. The Self Organizing Economy. Wiley-Blackwell, 1 edition. (1996).

[15] Sornette, D. Critical Phenomena in Natural Sciences: Chaos, Fractals, Self-organization and Disorder: Concepts and Tools (Springer Series in Synergetics). Springer, 2nd edition. (2006).

[16] Perc, M. Self-organization of progress across the century of physics. Scientific Reports, 31720 (2013).

[17] Locke, J., Second Treatise of Government: An Essay Concerning the True Original, Extent and End of Civil Government. John Wiley 85 Sons (2014) .

[18] Ostrom, E., Governing the commons. Cambridge University Press (2015).

[19] Axelrod, R., The Evolution of Cooperation (Revised Edition), Basic Books, (2006)

[20] Ostrom, E. Gardner, R. and Walker, J., Rules, games, and common-pool resources. University of Michigan Press (1994).

[21] Lakhani, K. R., and Wolf, R. G. Why hackers do what they do: Understanding motivation and effort in free/open source software projects. MIT Sloan Working Paper No. 4425-03 (2003).

[22] Roberts, J. A., Hann, I. and Slaughter, S.A., Understanding the motivations, participation, and performance of open source software developers: A longitudinal study of the Apache projects Management science $\mathbf{5 2 7}$ : 984-999 (2006).

[23] Maillart, T., Sornette, D., Spaeth, S. and von Krogh, G., Empirical tests of Zipfs law mechanism in open source Linux distribution, Physical Review Letters 10121 : 218701 (2008).

[24] Sen, R., Subramaniam, C. and Nelson, M. L., Determinants of the choice of open source software license. Journal of Management Information Systems 253 : 207-240 (2008).

[25] Lerner, J. and Tirole, J., The economics of technology sharing: Open source and beyond. Journal of Economic Perspectives 192, 99-120 (2005).

[26] Von Krogh, G., Haefliger, S., Spaeth, S., and Wallin, M. W., Carrots and rainbows: Motivation and social practice in open source software development. Mis Quarterly, 36(2), 649-676 (2012).

[27] Brooks, F.P., The mythical man-month. Addison-Wesley (1975).

[28] Chesbrough, HW. Open innovation: The new imperative for creating and profiting from technology. Harvard Business Press (2006).

[29] Tziner, A., Eden, D., Effects of Crew Composition on Crew Performance: Does the Whole 
Equal the Sum of Its Parts?, Journal of Applied Psychology 70 : 85-93 (1985).

[30] Sundstrom, E., De Meuse, K.P., Futrell, D., Work teams: Applications and effectiveness. American psychologist 45 : 120 (1990).

[31] Cohen, S.G., Bailey, D.E., What Makes Teams Work: Group Effectiveness Research from the Shop Floor to the Executive Suite, Journal of Management 23 : 239-290 (1997).

[32] Neuman, G.A., Wright, J., Team effectiveness: beyond skills and cognitive ability., Journal of Applied Psychology 84 : 376 (1999).

[33] Ennen, E., Richter, A., The Whole Is More Than the Sum of Its Parts Or Is It? A Review of the Empirical Literature on Complementarities in Organizations. Journal of Management 36 : 207-233 (2010).

[34] Lin, Y., Beyerlein, M.M., Communities of practice: A critical perspective on collaboration. Advances in Interdisciplinary Studies of Work Teams 12 : 53-79 (2006).

[35] Sacramento, C.A., Chang, M.W.S., West, M.A., Team innovation through collaboration, Advances in Interdisciplinary Studies of Work Teams 12 : 81-112 (2006).

[36] David, P. A., and Rullani, F., Dynamics of innovation in an open source collaboration environment: lurking, laboring, and launching FLOSS projects on SourceForge, Industrial and Corporate Change, 17(4), 647-710 (2008).

[37] Boehm, B.W., Software engineering economics, IEEE Trans Software Eng 10(1):421 (1984).

[38] Boehm, B.W., Clark, H., Brown, R., Chulani, MR, Steece, B., Software cost estimation with Cocomo II with Cdrom, 1st edn. Prentice Hall PTR, Upper Saddle River (2000).

[39] Levenshtein, V. I. . Binary codes capable of correcting deletions, insertions, and reversals. Soviet physics doklady 10(8),707-710 (1966).

[40] Cataldo, M., Wagstrom, P.A., Herbsleb, J.D. and Carley, K.M., Identification of coordination requirements: implications for the design of collaboration and awareness tools, In: Proceedings of the 2006 20th anniversary conference on computer supported cooperative work, CSCW 06, ACM, New York, pp 353 362. doi:10.1145/1180875.1180929 (2006)

[41] Gross, M., Haroche, S. ,Superradiance: An essay on the theory of collective spontaneous emission, Physics Reports 93: 301-396 (1982).

[42] Hawkes, A.G. and Oakes, D. (1974) A Cluster Process Representation of a Self-Exciting Process, Journal of Applied Probability 11 493-503.

[43] Mohler, G.O., Short, M.B., Brantingham, P.J., Schoenberg, F.P. and Tita, G.E., Self-Exciting 
Point Process Modeling of Crime. Journal of the American Statistical Association 106, 100108 (2011).

[44] Baldwin, A., Gheyas, I., Ioannidis, C., Pym, D. and Willams J., Contagion in Cybersecurity Attacks, In: Workshop on the Economics of Information Security, (2012).

[45] Aït-Sahalia, Y., Cacho-Diaz, J., and Laeven, R.J.A. Modeling Financial Contagion Using Mutually Exciting Jump Processes. Journal of Financial Economics 112 (3), 585-606 (2010).

[46] Filimonov, V. and Sornette, D., Quantifying reflexivity in financial markets: Toward a prediction of flash crashes, Physical Review E 85: 056108+ (2012).

[47] Filimonov, V., Bicchetti, D., Maystre, N., and Sornette, D. Quantification of the high level of endogeneity and of structural regime shifts in commodity markets. Journal of International Money and Finance 42 174-192 (2014).

[48] Maillart, T., Sornette, D., Frei, S., Duebendorfer, T., Saichev., A.I. Quantification of deviations from rationality with heavy tails in human dynamics, Physical Review E 83: 056101+ (2011)

[49] Daley, D.J., Vere-Jones, D. An introduction to the theory of point processes, Springer, 2nd edition (2003).

[50] Helmstetter, A., Sornette, D. Subcritical and supercritical regimes in epidemic models of earthquake aftershocks Journal of geophysical research 107 (B10): 2237 (2002).

[51] Helmstetter, A., Sornette, D. Importance of direct and indirect triggered seismicity in the ETAS model of seismicity, Geophys Res Lett 30 (11): 1576+ (2003).

[52] Saichev, A.I., Helmstetter, A., Sornette, D. Power-law distributions of offspring and generation numbers in branching models of earthquake triggering, Pure and Applied Geophysics 162: 1113-1134 (2005).

[53] Robles, G., Koch, S., and Gonzalez-Barahona J.M., Remote analysis and measurement of libre software systems by means of the CVSAnalY tool. In Proceedings of the 2nd ICSE Workshop on Remote Analysis and Measurement of Software Systems (RAMSS) 51-55 (2004).

[54] Hindle, A., German, D. M., and Holt, R., What do large commits tell us?: a taxonomical study of large commits. In Proceedings of the 2008 international working conference on Mining software repositories, 99-108 (2008).

[55] Alali, A., Kagdi, H., and Maletic, J. I., What's a typical commit? A characterization of open source software repositories. IIn: The 16th IEEE International Conference on Program 
Comprehension. ICPC 2008, pp 182191. (2008).

[56] Arafat, O., and Riehle, D., The commit size distribution of open source software, In: 42nd Hawaii International Conference on System Sciences HICSS'09, 1-8, (2009).

[57] Mandelbrot, B., and Taleb, NN.. Mild vs. wild randomness: focusing on risks that matter. Erscheint in: Diebold, F (2007).

[58] Saichev, A., Maillart, T., and Sornette, D., Hierarchy of temporal responses of multivariate self-excited epidemic processes. The European Physical Journal B, 86(4), 1-19 (2013).

[59] Keil, P., Principal agent theory and its application to analyze outsourcing of software development. In ACM SIGSOFT Software Engineering Notes 304, 1-5 (2005).

[60] Maillart, T., Zhao, M., Grossklags, J., and Chuang, J., Given enough eyeballs, all bugs are shallow? Revisiting Eric Raymond with bug bounty programs. Journal of Cybersecurity, 3(2), 81-90 (2017).

[61] Kuypers, M.A., Maillart, T. and Paté-Cornell, E., An Empirical Analysis of Cyber Security Incidents at a Large Organization. Stanford Working Paper (2016).

[62] Kuypers, M., and Maillart, T. Designing Organizations for Cyber Security Resilience, In: Workshop on the Economics of Information Security, WEIS'2018 (2018).

[63] Maillart, T., and Sornette, D. Using Prediction Markets to Incentivize and Measure Collective Knowledge Production arXiv preprint arXiv:1406.7746 (2014).

[64] Gulley, N., and Lakhani, K. R. The determinants of individual performance and collective value in private-collective software innovation. Harvard Business School Technology $\&$ Operations Mgt. Unit Working Paper No. 10-065. Available at SSRN: https://ssrn.com/abstract=1550352 (2010).

[65] Raymond, E. The cathedral and the bazaar. Knowledge, Technology \& Policy, 12(3), 23-49 (1999).

[66] Nosek, B. A., Alter, G., Banks, G. C., Borsboom, D., Bowman, S. D., Breckler, S. J., ... and Contestabile, M., Promoting an open research culture. Science, 348 (6242), 1422-1425 (2015).

[67] Munafò, M. R., Nosek, B. A., Bishop, D. V., Button, K. S., Chambers, C. D., du Sert, N. P., ... and Ioannidis, J. P. A manifesto for reproducible science. Nature Human Behaviour, 1(1), 0021 (2017).

[68] Maillart, T., and Sornette, D., Aristotle vs. Ringelmann: A response to Scholtes et al. on Superlinear Production in Open Source Software. arXiv preprint arXiv:1608.03608 (2016). 
[69] the size of the coordination is not clearly defined in the paper. We assume that the authors talked about the number of network edges in each time window of 7 days 